\title{
Strain effects on the surface optical transitions of GaAs
}

\author{
P. A. Postigo, * G. Armelles, and F. Briones \\ Instituto de Microelectrónica de Madrid (CNM-CSIC), Isaac Newton, 8, Parque Tecnológico de Madrid, \\ 28760 Tres Cantos, Madrid, Spain
}

(Received 30 April 1998)

\begin{abstract}
The surface optical properties of GaAs on GaP-strained layers have been analyzed in situ by chemical modulation spectroscopy. A clear surface-related optical transition has been identified, and the effect of strain on this transition has been studied, showing a blueshift of $30 \mathrm{meV}$ even for epitaxies as thin as $1 \mathrm{ML}$ of GaAs deposited on top of GaP. This small shift is consistent with a transition between dimer states and danglingbond states of the Ga-Ga dimers. [S0163-1829(98)07439-6]
\end{abstract}

Strain plays a major role in the growth mode of epitaxial films. In systems with a lattice mismatch between substrate and epitaxy, a layer-by-layer growth can only be obtained when the thickness of the overlayer is lower than a critical value. In this regime the strain developed in the overlayer can be very high. ${ }^{1,2}$ As a consequence, the surface electronic structure of the overlayer, and therefore the surface optical properties, may depend strongly on the lattice mismatch with the substrate. There have been some reports about the surface optical properties of strained epitaxial III-V compounds like InAs on GaAs. ${ }^{3,4}$ In these systems, a different surface reconstruction for the strained and nonstrained material was observed by reflection high-energy electron-diffraction (RHEED). Due to the strong relationship between reflectance difference spectra and surface reconstruction, ${ }^{5}$ the changes in the spectra could not be unambiguously related to the strain in the overlayer. Moreover, the spatial localization of the electronic states involved in the surface reflectance spectra is still under debate. Features related to transitions between surfacelike states are now interpreted as transitions between bulklike modified states. ${ }^{6-8}$ In order to identify these electronic transitions, the effect of introducing strain intentionally in the overlayer can help to distinguish between them, since the strain should affect the bulklike or surfacelike states in different ways.

In this Brief Report, we analyze the effect of the strain on the surface optical properties of GaAs. The strain is introduced in the GaAs layers by depositing several ML of GaAs on top of $\mathrm{GaP}$ using molecular-beam epitaxy (MBE). The lattice mismatch between these compounds is around $3.6 \%$. Recent studies have shown that the critical thickness for dislocation formation is of the order of $6 \mathrm{ML} .{ }^{9}$ Therefore, several monolayers of GaAs can be grown on top of GaP without strain relaxation. To analyze the effect of the strain, we have studied the changes in the reflectance spectra observed after deposition of 1-3 ML of GaAs on top of GaP. To monitor the surface optical properties, we have used the chemical modulation spectroscopy technique (CMS). ${ }^{10}$ This technique is based on the changing in the coverage of the last atomic layer, which induces a change in the intensity of the light reflected by the material $(\Delta R / R)$, that is recorded. The change in the chemistry of the surface is achieved by short pulses (during a time $t_{p}$ ) of a group-V molecular beam, using fast-acting, valved effusion cells for the group-V precursors inside the MBE reactor. If the temperature $T_{s}$ of the measured compound is sufficiently high (which is typically in the $450-580{ }^{\circ} \mathrm{C}$ range, depending on the compound) the deposition of a layer of group- $\mathrm{V}$ atoms is followed by its posterior desorption once the group- $\mathrm{V}$ cell is closed, during a time $t_{d}$. This modification of the surface chemistry produces a variation in the intensity of the reflected light, which is focused onto the substrate at near-normal incidence, passes through a polarizer, and is collected by a photodiode placed at the exit slit of a monochromator. The strongest changes in the intensity of the reflected light are observed when the light is collected linearly polarized along the direction parallel to the group-III dimers - typically [110] —whereas for the perpendicular direction the changes are much lower. The normalized change in the intensity is then plotted for each wavelength to obtain the spectrum of the compound $(1-3 \mathrm{eV})$. The change in the surface structure is monitored by RHEED through reconstruction.

In Fig. 1(a), we show the CMS spectrum of the GaP surface for light polarized parallel to the [110] direction. In this case $\sim 1-\mu \mathrm{m} \mathrm{GaP}$ layer was grown on a (001) GaP substrate. Atomic layer molecular beam epitaxy was used in the last 50

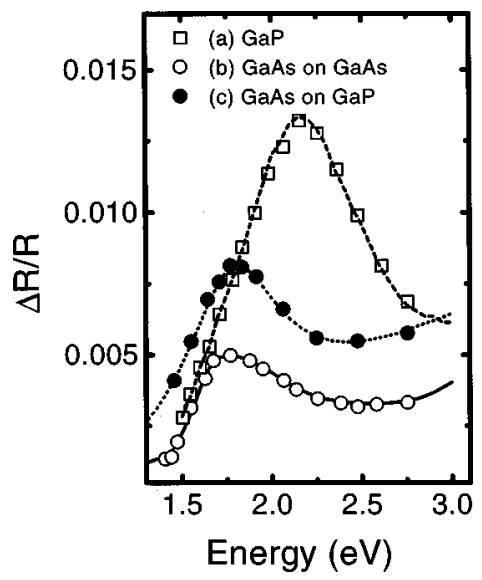

FIG. 1. CMS spectra of the different (001)-oriented layers measured for light polarized parallel to the [110] direction: (a) GaP on a GaP substrate, (b) GaAs deposited on a GaAs substrate, and (c) 1 $\mathrm{ML}$ of GaAs deposited on a GaP substrate. Lines show the fittings obtained using the $A$ and $B$ coefficients from Fig. 2, and the variations of the surface dielectric constants shown in Fig. 3. 


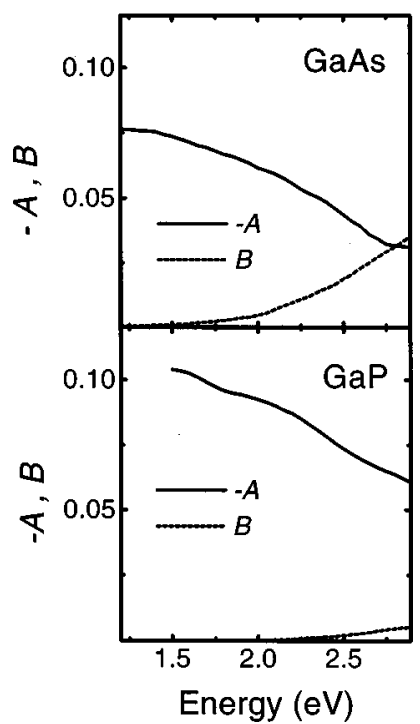

FIG. 2. $A$ and $B$ coefficients for GaAs and GaP in the measured range of energies, taken from Refs. 13 and 14.

ML, in order to enhance the flatness of the surface. ${ }^{11}$ The spectrum of this surface was obtained using a phosphorous beam $\left(\mathrm{P}_{2}\right)$ as the group $-\mathrm{V}$ element producing the chemical modulation on the surface. After closing the $\mathrm{P}_{2}$ cell $\left[t_{p}\right.$ $=0.2 \mathrm{~s}, x_{\mathrm{BEP}\left(\mathrm{P}_{2}\right)}=3 \times 10^{-6} \mathrm{mb}$ (where BEP denotes beam equivalent pressure)] the change from a $2 \times 2\left(\mathrm{P}_{2}\right.$-rich surface) to a $2 \times 4$ (Ga-rich surface) reconstruction takes about $t_{d}=2.5 \mathrm{~s}$ with a substrate temperature of $T_{s}=596{ }^{\circ} \mathrm{C}$. Figure 1 (b) shows the CMS spectrum of the GaAs surface $[\sim 1-\mu \mathrm{m}$ GaAs grown on a GaAs (001) substrate] for light analyzed along the [110] direction, parallel to the Ga dimers. In this case, the chemical modulation of the surface is achieved by pulsing the $\mathrm{As}_{2}$ beam, using similar BEP and adsorption and desorption times $t_{p}$ and $t_{d}$, and with a substrate temperature of $T_{s}=556{ }^{\circ} \mathrm{C}$. The observed reconstruction patterns are now $2 \times 4$ (As-rich) and $4 \times 2$ (Ga-rich) surfaces. Using athree layer model (vacuum, surface layer and bulk), ${ }^{12}$ the changes in the reflectance can be related to the changes in the surface dielectric constant through the expression

$$
\Delta R / R=\left(R_{\mathrm{III}}-R_{\mathrm{V}}\right) / R_{\mathrm{III}}=(-8 \pi d / \lambda)\left(A \Delta \varepsilon_{\mathrm{surf}}^{i}+B \Delta \varepsilon_{\mathrm{surf}}^{r}\right),
$$

where $\Delta \varepsilon_{\text {surf }}^{r}$ and $\Delta \varepsilon_{\text {surf }}^{i}$ are the changes in the real and imaginary part of the surface dielectric constant $\varepsilon_{\text {surf }}$, produced by the change in the coverage of the surface, and they are defined by

$$
\begin{aligned}
& \Delta \varepsilon_{\text {surf }}^{r}=\varepsilon_{\text {surf III }}^{r}-\varepsilon_{\text {surf V }}^{r}, \\
& \Delta \varepsilon_{\text {surf }}^{i}=\varepsilon_{\text {surf III }}^{i}-\varepsilon_{\text {surf V }}^{i} .
\end{aligned}
$$

$d$ is the thickness of the surface layer. The coefficients $A$ and $B$ depend on the bulk properties of GaAs and GaP, and they are plotted in Fig. 2 for the $1-3-\mathrm{eV}$ range. These $A$ and $B$ coefficients have been calculated using the measured dielectric constants of GaAs (Ref. 13) and GaP. ${ }^{14}$ As shown, the changes in the reflectivity are mainly determined by the changes in the imaginary part of the surface dielectric constant. In Fig. 3 we show the variation with the energy of the

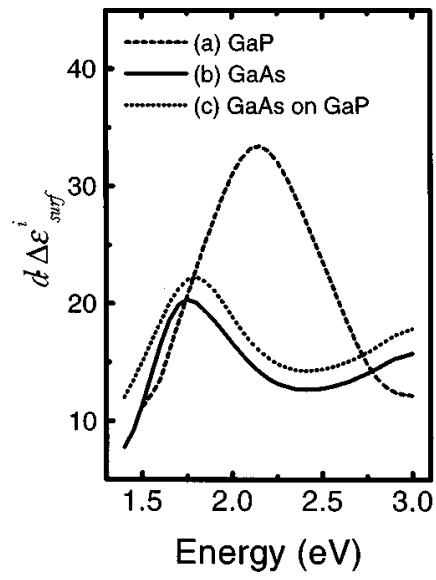

FIG. 3. Variation of the imaginary part of the surface dielectric constant in the [110] direction with the energy, obtained from the fitting to the experimental spectra of Fig. 1. (a) GaP. (b) GaAs on GaAs. (c) 1 ML of GaAs on GaP.

imaginary part of the surface dielectric constants, $\Delta \varepsilon_{\text {surf }}^{i}$, for $\mathrm{GaP}$ - line (a) — and GaAs_-line (b) — based on fitting to the experimental spectra of Figs. 1(a) and 1(b) respectively, and multiplied by the thickness of the layer, $d$. A well-defined peak, clearly located at a different energy $(2.18 \mathrm{eV}$ for $\mathrm{GaP}$ and $1.77 \mathrm{eV}$ for $\mathrm{GaAs}$ ) is observed for each compound. To analyze the effect of the strain on the GaAs spectrum, we have measured the CMS spectrum after the deposition of one atomic monolayer of GaAs on top of GaP. The modulation of the surface coverage of this GaAs monolayer is achieved by pulsing the $\mathrm{As}_{2}$ beam, with $t_{d}, t_{p}$, and BEP parameters similar to those for the measurement of GaAs on $\mathrm{GaAs}$, and at the same substrate temperature $T_{s}=570{ }^{\circ} \mathrm{C}$. During the measurement, RHEED showed the same reconstruction patterns than in the measurement of GaAs on GaAs. The result is shown in Fig. 1(c). The CMS spectrum of $1 \mathrm{ML}$ of GaAs on GaP presents a well-defined peak at an energy similar to the GaAs-on-GaAs peak. The intensity of the peak is higher than in the GaAs-on-GaAs measurement, mainly due to the different values of the $A$ and $B$ parameters for the respective substrate layers GaAs and GaP. We have obtained the same CMS spectrum for a GaAs coverage up to 3 ML. At a higher amount of deposited GaAs, we have observed a change in the RHEED pattern, which becomes slightly spotty, indicating a two- to three-dimensional change in the surface structure. For coverage higher than $3 \mathrm{ML}$, the intensity of the spectrum decreases strongly. This effect can be due to the formation of $\mathrm{GaAs}$ facets oriented so that $\mathrm{Ga}$ dimers might not form. ${ }^{15}$ Figure 3(c) shows the variation of the imaginary part of the dielectric constant for GaAs on $\mathrm{GaP}$ vs energy, obtained from the fitting to the experimental spectrum. There is a striking similarity between the positions and shapes of the imaginary part of the dielectric constant for GaAs deposited on GaP and GaAs deposited on GaAs.

Two main conclusions can be drawn: the changes observed in the surface dielectric constant are mainly due to transitions related to electronic states strongly localized in the very first surface layers, i.e., surface-like states. The second conclusion is that, despite the fact that there is a high lattice mismatch of $3.6 \%$ between $\mathrm{GaAs}$ and $\mathrm{GaP}$, the energy position of the transition observed is not very significantly 
affected by the strain-it shifts $30 \mathrm{meV}$ toward higher energies-much less than what is observed in bulklike transitions. For example, the $E_{1}$ transition, which is located in the energy range studied here, shifts $99 \mathrm{meV}$ for an in-plane strain of $3.6 \%$. Due to the strong spatial localization of the electronic states involved in the transition, the surface dimer can be simulated as two Ga atoms bounded to four As atoms localized in a lower plane, and using a simple tight-binding model the effect of the strain can be estimated. From the results of Ref. 1, we can consider that the GaAs overlayer suffers an in-plane compression of $3.6 \%$, and assuming that the distances and bond angles are modified by the strain according to the elastic theory, ${ }^{1}$ a $3.6 \%$ in-plane compression in the $\mathrm{As}_{2}=\mathrm{Ga}-\mathrm{Ga}=\mathrm{As}_{2}$ molecule produces a shift of the dimer-dangling-bond transition of $20 \mathrm{meV}$. We have used the tight-binding parameters of Ref. 16 for $\mathrm{Ga}$ and $\mathrm{As}$, and the Ga-As and Ga-Ga interactions, and Ref. 17 for the As-As interaction parameters. We have scaled those interactions and the strain-induced changes in the parameters according to the scaling laws of Refs. 16 and 18, that give a very good

*Present address: Massachusetts Institute of Technology, 77 Mass. Ave., Room 13-3062, Cambridge, Massachusetts 02139. Fax: +1 617258 6640. Electronic address: aitor@ oevlsi.mit.edu

${ }^{1}$ R. E. Martinez, W. M. Augustyniak, and J. A. Golovchenko, Phys. Rev. Lett. 64, 1035 (1990).

${ }^{2}$ A. J. Schell-Sorokin and R. M. Tromp, Phys. Rev. Lett. 64, 1039 (1990).

${ }^{3}$ S. M. Scholz, A. B. Muller, W. Richter, D. R. T. Zahn, D. I. Westood, D. A. Woolf, and R. H. Williams, J. Vac. Sci. Technol. B 10, 1710 (1992).

${ }^{4}$ R. Arès, C. A. Tran, and S. P. Watkins, Appl. Phys. Lett. 67, 1576 (1995).

${ }^{5}$ I. Kamiya, D. E. Aspnes, L. T. Florez, and J. P. Harbison, Phys. Rev. B 46, 15894 (1992).

${ }^{6}$ D. E. Aspnes, Y. C. Chang, A. A. Studna, L. T. Florez, H. H. Farell, and J. P. Harbison, Phys. Rev. Lett. 64, 192 (1990).

${ }^{7}$ K. Uwai and N. Kobayashi, Phys. Rev. Lett. 78, 959 (1997).

${ }^{8}$ T. Yasuda, L. Mantese, U. Rossow, and D. E. Aspnes, Phys. Rev. Lett. 74, 3431 (1995).

${ }^{9}$ J. A. Prieto, G. Armelles, M. E. Pistol, P. Castrillo, J. P. Silveira, and F. Briones, Appl. Phys. Lett. 70, 3449 (1997). description of the deformation potentials of III-V compounds. This calculated shift is similar to the experimentally found $[30 \mathrm{meV}$, obtained from the fittings showed in Figs. 3(a) and 3(c)]. Our results support the idea from Aspnes and co-workers ${ }^{6,19}$ that the peak observed in the imaginary part of the GaAs surface dielectric constant is associated with a $\mathrm{Ga}$ dimer-related transition involving dimer states and dangling-bond states. This group-III dimer transition is likely responsible for the peaks found in other III-V compounds. $^{20,21}$ In conclusion, we have shown that surface Ga dimer produces an optical transition, with a strong localization in the first atomic layer. The dependence of this transition on the strain is much lower than the dependence observed in bulklike transitions occurring in the energy range measured. The observed small shift to higher-energy values is also consistent with a transition occurring between dimer and dangling-bond states of the Ga dimers.

This work was partially supported by a CICYT MAT950966-c02-01 Research Action.

${ }^{10}$ P. A. Postigo, T. Utzmeier, G. Armelles, and F. Briones, J. Cryst. Growth 175/176, 298 (1997).

${ }^{11}$ F. Briones, L. González, and A. Ruiz, Appl. Phys. A: Solids Surf. 49, 729 (1989).

${ }^{12}$ J. D. E. McIntyre and D. E. Aspnes, Surf. Sci. 24, 417 (1971).

${ }^{13}$ P. Lautenschlager, M. Garriga, S. Logothetidis, and M. Cardona, Phys. Rev. B 36, 4813 (1987).

${ }^{14}$ S. Zollner, M. Garriga, J. Kircher, J. Humlicek, M. Cardona, and G. Neuhold, Phys. Rev. B 48, 7915 (1993).

${ }^{15}$ M. A. Herman and H. Sitter, Molecular Beam Epitaxy, Fundamentals and Current Status, 2nd ed. (Springer, Berlin, 1996).

${ }^{16}$ C. Priester, G. Allan, and M. Lanoo, Phys. Rev. B 37, 8519 (1988).

${ }^{17}$ P. K. Larsen et al. Phys. Rev. B 26, 3222 (1982).

${ }^{18}$ M. C. Muñoz and G. Armelles, Phys. Rev. B 48, 2839 (1993).

${ }^{19}$ D. E. Aspnes, J. P. Harbison, A. A. Studna, and L. T. Florez, Phys. Rev. Lett. 59, 1687 (1987).

${ }^{20}$ P. A. Postigo, G. Armelles, T. Utzmeier, and F. Briones, Phys. Rev. B 57, 1359 (1998).

${ }^{21}$ P. A. Postigo, G. Armelles, T. Utzmeier, and F. Briones, Phys. Rev. B 57, 1362 (1998). 